\title{
ON VECTOR FIELDS IN THE PLANE WITH THE REFLECTION PROPERTY
}

\section{S. BERHANU AND J. HOUNIE}

Abstract. We discuss necessary and sufficient conditions for the validity of the analogue of the Schwarz Reflection Principle for homogeneous solutions of complex vector fields in the plane.

\section{INTRODUCTION}

The classical Schwarz Reflection Principle [S] may be stated as follows:

- Let $\gamma_{1}$ and $\gamma_{2}$ be real analytic curves in $\mathbb{C}$. Then every holomorphic function $f(z)$ defined on a side of $\gamma_{1}$ that extends continuously up to $\gamma_{1}$ and maps $\gamma_{1}$ into $\gamma_{2}$ has a holomorphic extension across $\gamma_{1}$.

Generalizations of this reflection principle in higher dimensions have been the subject of study in numerous papers including $[\mathrm{BJT},[\mathrm{BHR}, \overline{\mathrm{BR}},[\mathrm{DF}, \overline{\mathrm{EH}}], \mathrm{Hu} 1$, $[\mathrm{Me}$, and we refer the reader to the book [BER] and the survey article $\mathrm{Hu} 2$ for a more detailed account.

In this work we explore the validity of the analogue of the classical Schwarz Reflection Principle for solutions of the homogeneous equation of locally integrable, smooth complex vector fields

$$
L=a(x, y) \frac{\partial}{\partial y}+b(x, y) \frac{\partial}{\partial x}
$$

on a domain $\Omega$ in the plane where we will require that the coefficients $a(x, y)$ and $b(x, y)$ have no common zeros. The local integrability of $L$ means that for each $p \in \Omega$, there is a smooth function $Z(x, y)$ defined on a neighborhood $\Omega_{p}$ of $p$ that satisfies $L Z=0$ and the differential $d Z \neq 0$ (on the subject of locally integrable vector fields we refer the reader to $[\mathrm{T}]$ and $[\mathrm{BCH}]$ ). The function $Z$ is called a first integral of $L$ on $\Omega_{p}$. Given a smooth curve $\gamma$ in $\Omega$ and a point $p \in \gamma$, we will consider a function $u$ that is defined on a side of $\gamma$, is continuous up to $\gamma$ near $p$, satisfies the equation $L u=0$ on that side of $\gamma$ and maps $\gamma$ into a real analytic curve. If every such $u$ has a continuous extension $w$ that is a solution of $L w=0$ in a full neighborhood of $p$, we will say that $\gamma$ has the reflection property for $L$ at the point $p$.

In general, as the following example shows, the reflection property may not hold:

Example. Let

$$
L=\frac{\partial}{\partial y}+i h(y) \frac{\partial}{\partial x}
$$

Received by the editors April 26, 2011 and, in revised form, December 4, 2011.

2010 Mathematics Subject Classification. Primary 35B60, 35F05; Secondary 30B40, 35H10.

Key words and phrases. Prolongation of solutions, reflection property.

This work was supported in part by NSF DMS 1001283, CNPq, FAPESP and CAPES.

(C)2013 American Mathematical Society 
where

$$
h(y)=0, \text { for } y \geq 0 \text { and } h(y)=e^{\frac{1}{y}} \text { when } y<0 .
$$

If $u(x, y)=u_{0}(x)$ is any real-valued continuous function, $u(x, y)$ is a homogeneous solution when $y>0$, but it may not be extendable to a solution in the region $y<0$ since the analytic wave-front set of the boundary value of a solution defined on $y<0$ misses a direction. Note that $L$ is a smooth, locally solvable vector field.

On the other hand, this kind of behavior cannot occur if $L$ has real analytic coefficients, even for solutions of the semilinear equation. Indeed, one of the results we prove in this work is

Theorem. Let $L$ be a real analytic vector field in a neighborhood of the origin and suppose $\gamma$ is a real analytic, noncharacteristic curve through the origin that divides a disc $D$ centered at $(0,0)$ into two regions $D^{+}$and $D^{-}$. Let $f(x, y, \zeta)$ be a real analytic function that is holomorphic in $\zeta$. Then if $u(x, y)$ is a continuous function on $\overline{D^{+}}$which is a solution of $L u=f(x, y, u)$ in $D^{+}$, and $u(\gamma)$ lies in a real analytic curve $\Gamma$, then $u$ extends as a solution of the same equation in a neighborhood of the origin.

Among the locally integrable vector fields, the ones whose solutions enjoy the best regularity properties are those that are called hypocomplex vector fields. A vector field $L$ is called hypocomplex if every solution of $L u=0$ has the local form $u=H \circ Z$ where $H$ is holomorphic and $Z$ is a first integral of $L$. We note that a vector field is hypocomplex if and only if every first integral is a local homeomorphism. In Section 1 we will show that if a smooth curve $\gamma$ has the reflection property for a hypocomplex vector field $L$ and $Z$ is a first integral defined near a point $p \in \gamma$, then $Z(\gamma)$ is a real analytic curve. Motivated by this observation, in the same section we will introduce what we call $L$-analytic curves for a locally integrable vector field $L$. To a certain extent, $L$-analytic curves play the role that real analytic curves do in the classical reflection principle, but, in contrast with the classical situation, additional ingredients must be brought into play in order to determine when the reflection property holds.

In Section 2 we will present a necessary and sufficient condition for a noncharacteristic $L$-analytic curve of a smooth locally integrable vector field to have the reflection property. In the same section we will prove the theorem stated above for the semilinear equation $L u=f(x, y, u)$.

In Section 3 we study the reflection property across characteristic curves. For a class of characteristic, $L$-analytic curves $\gamma$-where $L$ is a smooth locally integrable vector field - we will present a necessary and sufficient condition that $\gamma$ should satisfy in order to have the reflection property for $L$.

The authors are grateful to Bernhard Lamel for suggesting the problem considered in this paper. They also wish to express their appreciation to the referee whose thorough reading of the manuscript, corrections and comments have resulted in an improved version of our paper.

\section{A Class of CURVES}

In the classical Schwarz Reflection Principle for holomorphic functions, if a side of a smooth curve $\gamma$ has the reflection property for the Cauchy Riemann operator, then $\gamma$ has to be a real analytic curve. We first wish to understand the smooth curves that have the reflection property for hypocomplex vector fields. In the 
following propositions, we assume that the reflection property holds either on one side or both sides of the curve.

Proposition A. Let $L$ be a real analytic, hypocomplex vector field and suppose $\gamma$ is a $C^{\infty}$ curve through the origin with the reflection property on one side at the origin. Then $\gamma$ is analytic and for any first integral $Z$ of $L$ defined near the origin, $Z(\gamma)$ is real analytic near $Z(0,0)$. As a consequence, the reflection property holds on both sides of $\gamma$. Conversely, if $Z(\gamma)$ is a real analytic curve, then $\gamma$ has the reflection property.

Proof. Without loss of generality, we may assume that in a neighborhood of the origin, $L=\frac{\partial}{\partial y}+a(x, y) \frac{\partial}{\partial x}$ for some real analytic function $a(x, y)$. Let $Z(x, y)$ be a real analytic function that is a solution of the Cauchy problem $L Z=0, Z(x, 0)=x$ near the origin. After changing coordinates $x^{\prime}=\Re Z(x, y), y^{\prime}=y$ and dropping the primes, we get a real analytic first integral $Z=x+i \varphi(x, y)$ with $\varphi(x, y)$ realvalued and $\varphi(x, 0) \equiv 0$. Since the proposition holds for elliptic vector fields, we may also assume that $\varphi_{y}(0,0)=0$. Assume first that $\gamma$ is characteristic to $L$ at the origin. Then the curve $\gamma$ has the form $\gamma(s)=(x(s), s)$ for some smooth function $x(s), x(0)=0$ and $x^{\prime}(0)=0$. By hypocomplexity, $Z$ is a homeomorphism of a disc $D$ centered at 0 which is divided into two components $D^{+}$and $D^{-}$. Assume the reflection property for the side $D^{+}$. Let $\sigma=Z(\gamma \cap D)$. By the Riemann mapping theorem, we can find a holomorphic function $H$ on $Z\left(D^{+}\right)$continuous up to $\sigma$, mapping $\sigma$ into the reals with $H(0)=0, H\left(Z\left(D^{+}\right)\right)$contained in the upper half plane.

Since $w=H \circ Z$ satisfies $L w=0$ on $D^{+}$, is continuous up to $\gamma \cup D$, and is realvalued on $\gamma \cup D$, and since $\gamma$ has the reflection property, it extends as a solution to a neighborhood of $(0,0)$. By hypocomplexity, $w=\tilde{H} \circ Z$ near $(0,0)$ for some holomorphic function $\tilde{H}$. Since $Z$ is a homeomorphism, $\tilde{H}$ and $H$ agree on an open set with $(0,0)$ on its boundary. So they coincide in a neighborhood of $(0,0)$. Thus, $H$ extends as a holomorphic function in a neighborhood of the origin. We will denote the extension by $H$.

Suppose first that both $x(s)$ and $\varphi(x(s), s)$ vanish at the origin to finite order. Then the curve $\sigma$ can be expressed in the form $\sigma(s)=\left(a(s) s^{k}, b(s) s^{n}\right)$ for some nonnegative integers $n, k \geq 2, a(0) \neq 0$ and $b(0) \neq 0$. Write $\sigma=\sigma^{+} \cup \sigma^{-}$, where $\sigma^{+}$denotes the part for $s \geq 0$ and $\sigma^{-}$the part for $s \leq 0$. Note that since $Z$ is a homeomorphisn, these two arcs meet only at the origin, and they intersect at an angle of either $\pi$ or zero radians. If $x(s)$ vanishes to a finite order at the origin, we also reach the same conclusion about this latter angle of intersection. Suppose now that $x(s)$ vanishes to infinite order at the origin. Writing $\zeta(s)=\varphi(x(s), s)$, we then see that

$$
\frac{d^{k} z}{d s^{k}}(0)=\frac{\partial^{k} \varphi}{\partial y^{k}}(0,0) \text { for all } k
$$

and so since $\varphi(0, y)$ does not vanish identically (by hypocomplexity and analyticity), we conclude that $\zeta(s)=\varphi(x(s), s)$ vanishes to a finite order at the origin, and so the angle of intersection between the two arcs is still zero or $\pi$. Since $H$ is a nonzero function, there exists a biholomorphism $G$ with $G(0)=0$ and an integer $m$ such that $H(z)=G(z)^{m}$ for $z$ in a neighborhood of the origin. Since $G(\sigma(s))^{m} \in \mathbb{R}$ for all $s$ (say $|s|<\epsilon), G\left(\sigma^{+}(s)\right) \subset \Gamma_{1}$ and $G\left(\sigma^{-}(s)\right) \subset \Gamma_{2}$, where $\Gamma_{1}$ and $\Gamma_{2}$ are rays through the $m^{\text {th }}$ roots of unity and -1 . Since $G$ preserves the angle between $\sigma^{+}$ 
and $\sigma^{-}$at the origin and the $\Gamma_{j}$ are distinct, it follows that their union forms a line through the origin which proves that $\sigma$ is a real analytic curve.

Let $v(z)=\operatorname{Im} H(z)$. Then the harmonic function $v(z)$ is positive on the domain $Z\left(D^{+}\right)$and $v(0)=0$. Moreover, since $Z\left(D^{+}\right)$has a $C^{2}$ boundary at the origin, it satisfies the interior sphere condition there, and hence by Hopf's Lemma, the differential $d v(0) \neq 0$. It follows that $H$ is a biholomorphism on a neighborhood of the origin. It also follows that $\gamma$ has the reflection property on both sides.

We will next show that $x(s)$ and hence $\gamma$ are real analytic. We know that $\{x(s)+i \varphi(x(s), s)\}$ is contained in an analytic curve $\sigma$. Assume first that near the origin, $\sigma$ is defined by $y=f(x), f(0)=0, f$ real analytic. If $f$ vanished identically, we would have $Z(x(s), s) \equiv x(s)$, but since $Z(x, 0) \equiv x$, this would contradict the fact that the map $Z$ is a homeomorphism. Then consider the real analytic function $q(x, s)=\varphi(x, s)-f(x)$. We know that $q(0,0)=0$ and $q(x, 0)=-f(x)$ does not vanish identically. Therefore, by the Weierstrass Preparation Theorem, there is a polynomial $p(x, y)=x^{n}+b_{n-1}(y) x^{n-1}+\cdots+b_{0}(x)$ such that for $s$ near 0 , $q(x(s), s)=p(x(s), s)=0$. By Proposition 5.5.19 in BER], we conclude that $x(s)$ is a real analytic function. Assume next that $\sigma$ near the origin is given by $x=h(y)$, $h(0)=0, h$ real analytic. Then $r(x(s), s)=0$, where $r(x, s)=x-h(\varphi(x, s))$ is real analytic with $r_{x}(0,0) \neq 0$. By the implicit function theorem, we conclude that $x(s)$ is real analytic.

Suppose now $\gamma$ is noncharacteristic to $L$ at the origin, in which case it has the form $\gamma(s)=(s, y(s))$ for some smooth function $y(s)$ with $y(0)=0$. If $\gamma$ has the reflection property for $L$ on a side of $\gamma$, then the smooth curve $\sigma(s)=Z(\gamma(s))=$ $s+i \varphi(s, y(s))$ will have the reflection property for holomorphic functions and so $\sigma(s)$ has to be real analytic. But then, since $\varphi(0, y)$ does not vanish identically, as we saw already, the analyticity of $\varphi(s, y(s))$ implies that $y(s)$ is real analytic.

The converse is easy to see.

Proposition B. Let $L$ be a $C^{\infty}$, hypocomplex vector field and suppose $\gamma$ is a $C^{\infty}$ curve through the origin with the reflection property on both sides at the origin. Then for any first integral $Z$ of $L$ near the origin, $Z(\gamma)$ is real analytic near $Z(0,0)$. Conversely, if $Z(\gamma)$ is real analytic, then $\gamma$ has the reflection property.

Proof. Let $Z=x+i \varphi(x, y)$ be a smooth first integral of $L$, with $\varphi(0,0) \equiv 0$, $\varphi_{y}(0,0)=0$, and $\varphi$ real-valued. Assume first that $\gamma$ is characteristic to $L$ at the origin. The curve $\gamma$ has the form $\gamma(s)=(x(s), s)$ for some smooth function $x(s)$, $x(0)=0$ and $x^{\prime}(0)=0$. By hypocomplexity, $Z$ is a homeomorphism of a disc $D$ centered at 0 which is divided into two components $D^{+}$and $D^{-}$by $\gamma$. Let $\sigma=Z(\gamma \cap D)$. By the Riemann Mapping Theorem, we can find a holomorphic function $H$ on $Z\left(D^{+}\right)$continuous up to $\sigma$, mapping $\sigma$ into the reals with $H(0)=0$, $H\left(Z\left(D^{+}\right)\right)$contained in the upper half plane. Likewise we can find a holomorphic function $P(z)$ on $Z\left(D^{-}\right)$continuous up to $\sigma$, mapping $\sigma$ into the reals with $P(0)=$ $0, P\left(Z\left(D^{-}\right)\right)$contained in the upper half plane. Since $\gamma$ has the reflection property on both sides, $H$ and $P$ extend as holomorphic functions on a neighborhood of the origin. We will denote the extensions by $H$ and $P$. Let $H(z)=G(z)^{m}$, where $G$ is a biholomorphism with $G(0)=0$. We know that $G(\sigma) \subset \Gamma_{1} \cup \Gamma_{2}$, which are rays through the $m^{\text {th }}$ roots of 1 and -1 . Let $W$ be a disc centered at the origin such that $W \Subset Z(D)$, and set $W^{+}=W \cap Z\left(D^{+}\right), W^{-}=W \cap Z\left(D^{-}\right)$. Observe that either $G\left(W^{+}\right)$or $G\left(W^{-}\right)$satisfies the interior sphere condition at the origin. Say $G\left(W^{+}\right)$ 
satisfies this condition. Let $v(z)=\operatorname{Im} H \circ G^{-1}(z)$ so $v$ is harmonic, and if $B$ is an open ball in $G\left(W^{+}\right)$with $0 \in \partial B$, then $v$ is smooth up to the boundary of $B$, and positive inside the ball. Since $v(0)=0$, by Hopf's Lemma, the differential of $v$ at the origin is nonzero and so $H$ is a biholomorphism at the origin. We conclude that $\gamma$ is $L$-analytic. If $G\left(W^{-}\right)$is the one with the interior sphere condition, we reason with $\operatorname{Im} P \circ G^{-1}(z)$ on the domain $G\left(W^{-}\right)$to reach the same conclusion about $\gamma$. If $\gamma$ is noncharacteristic to $L$ at the origin, as we saw in the preceding proposition, $Z(\gamma)$ is a real analytic curve. If $Z(\gamma)$ is a real analytic curve, it easily follows that $\gamma$ has the reflection property.

The following example shows that in general, unlike in the analytic case, for a smooth hypocomplex vector field, a smooth curve may satisfy the reflection property for solutions defined only on one side.

Example. Set $x(s)=e^{-1 / s^{2}}, Z(x, t)=x+i \varphi(t), \varphi(t)=s n(t) a e^{-1 / t^{2}}$, where $s n(t)$ is equal to +1 for $t>0$, equal to -1 for $t<0$ and equal to 0 at 0 and $a>0$. Then $\varphi(t)$ is strictly increasing and $Z(x, t)$ is the smooth first integral of a hypocomplex vector field. The curve $\gamma(s)=(x(s), s)$ is smooth and it is characteristic precisely at the origin.

Let $\sigma(s)=Z(\gamma(s))$ so $\sigma(s)=e^{-1 / s^{2}}(1+i \operatorname{sn}(s) a)$, and $\sigma^{+}$is contained in the ray along the direction $(1, a)$ while $\sigma^{-}$is contained in the ray along the direction $(1,-a)$. The angle $\theta$ between the two rays is $2 \arctan a$, which can be anything between 0 and $\pi$. In particular $\sigma$ has a corner at the origin. The curve $\gamma$ divides any disc $D$ centered at the origin into two components $D^{+}$and $D^{-}$, where $D^{+}$will denote the region contained in the right half plane. The map $Z$ is a homeomorphism from $D$ onto an open set $U$; let $U^{+}=Z\left(D^{+}\right)$and $U^{-}=Z\left(D^{-}\right)$. Observe that $\gamma$ has the reflection property for $L$ for solutions defined on the side $D^{+}$(respectively $D^{-}$) if and only if $\sigma$ has the reflection property for holomorphic functions defined on $U^{+}$(respectively $U^{-}$). The latter holds for $U^{+}$if and only if

$$
2 \arctan a \in\left\{\frac{\pi}{m}: m=1,2,3, \ldots\right\} .
$$

Moreover, when $2 \arctan a=\pi / m$ for some $m \in \mathbb{N}$, then $\sigma$ will not have the reflection property for holomorphic functions defined on $U^{-}$, and hence $\gamma$ will not have the reflection property for solutions of $L$ defined on the side $D^{-}$.

The preceding results motivate the following concept:

Definition 1.1. Let $\gamma:(0,1) \longrightarrow \Omega \subset \mathbb{R}^{2}$ be a smooth curve and assume that $L$ is a smooth locally integrable vector field defined on $\Omega$. We say that $\gamma$ is $L$ analytic if for every $s_{0} \in[0,1]$ there exists a local first integral $Z(x, t)$ defined on a neighborhood of $\gamma\left(s_{0}\right)$ and an open real analytic arc $\Gamma$ such that $Z(\gamma(s)) \subset \Gamma$ for $s$ close to $s_{0}$.

Suppose that $u$ is a continuous function that satisfies $L u=0$ on one side of an $L$-analytic curve $\gamma$. We will be interested in extending $u$ across $\gamma$ as a continuous homogeneous solution of $L$. In doing so, we may reason locally, and look for possible extensions of $u$ across $\gamma$ on a neighborhood of a given point $\gamma\left(s_{0}\right)$. It may happen that, for $s$ close to $s_{0}, Z \circ \gamma(s)$ reduces to a point. In this case, $\gamma$ is trivially $L$ analytic and also $\gamma(s)$ will be a characteristic curve for $L$ at any one of its points 
close to $\gamma\left(s_{0}\right)$. The other possibility is that no open subarc of $\gamma$ containing $s_{0}$ is mapped by $Z$ into a point.

Note that if $Z \circ \gamma(s) \subset \Gamma$ for $s$ close to $s_{0}$ with $\Gamma$ real analytic, and $Z\left(\gamma\left(s_{0}\right)\right)$ is an interior point of $Z(D)$ for any neighborhood $D$ of $\gamma\left(s_{0}\right)$, then it follows from a standard argument that for any other first integral $W$ defined on a neighborhood of $\gamma\left(s_{0}\right)$, there is a biholomorphic map $G$ such that $W$ may be written as $W=G \circ Z$ on a neighborhood of $\gamma\left(s_{0}\right)$. Hence, $W \circ \gamma(s)$ will be contained for $s$ close to $s_{0}$ in the real analytic arc $G(\Gamma)$. This situation always occurs when $L$ is hypocomplex because all local first integrals of such $L$ are open maps; this includes the case of elliptic vector fields. In general, however, a curve $\gamma(s)$ may be $L$-analytic but $W \circ \gamma(s)$ may fail to be real analytic for some other first integral $W$; for instance, if $L=\partial_{t}, Z=x$ and $W=x+i g(x)$ are first integrals of $L$ where we choose $g(x)$ to be a smooth function that is not real analytic. If $\gamma(s)=(s, 0)$, we see that $Z \circ \gamma(s)=(s, 0)$, while $W \circ \gamma(s)$ is not contained in an analytic arc.

Examples. (1) If $L$ has real analytic coefficients we may always choose a real analytic first integral of $L$ which in appropriate real analytic coordinates is written as $Z(x, t)=x+i \varphi(x, t)$ on a neighborhood $D$ of a given point $p$. Thus, any real analytic noncharacteristic curve $\gamma(s)$ is $L$-analytic, since nearby $p$ it may be parametrized as $\gamma(s)=(s, t(s))$ and will be mapped by $Z$ into the real analytic curve $\Gamma(s)=(s, \varphi(s, t(s)))$. Conversely, assume that $L$ is not a multiple of a real vector field and $\gamma$ is a noncharacteristic $L$-analytic curve passing through $p$ and for some analytic curve $\Gamma$ and some analytic first integral $W, W(\gamma) \subset \Gamma$ as sets. Then $\gamma$ must be analytic. To see this, first note that since both $Z$ and $W$ are real analytic first integrals, there is a biholomorphism $H$ defined near $Z(p)$ such that $W=H \circ Z$ near $p$ which implies that $Z(\gamma)$ is contained in a real analytic curve. Let $\gamma(s)=(s, h(s))$. Then there is a real analytic function $f$ such that $\varphi(s, h(s))-f(s)=0$. If $\varphi(0, y)$ is not identically zero, we can use the Weierstrass Preparation Theorem and Proposition 5.5.19 in [BER] as in the proof of Proposition A to conclude that $h(s)$ and hence $\gamma(s)$ is real analytic. If $\varphi(0, y) \equiv 0$, since $L$ is not a multiple of a real vector field, we can find a positive integer $m$ and a real analytic function $\psi(x, y)$ such that $\varphi(x, y)=x^{m} \psi(x, y)$ and $\psi(0, y)$ does not vanish identically. We can then argue as before to conclude that $\gamma(s)$ is real analytic. If $W$ is just smooth we may write - after shrinking $D-W=\Psi \circ Z$, with $\Psi$ holomorphic in the interior of $Z(D)$. Thus, if $Z(p)$ is an interior point of $Z(D), W$ will be analytic on a neighborhood of $p$ and $\gamma$ will be analytic on a neighborhood of $p$.

(2) Consider the Grushin vector field $L=\partial_{t}-i 6 t^{5} \partial_{x}$ that is elliptic for $t \neq 0$ and has a real analytic first integral $Z(x, t)=x+i t^{6}$. The curve $\gamma(s)=\left(s^{3}, s\right)$ is characteristic to $L$ at the origin and it is $L$-analytic since $Z(\gamma(s))=s^{3}+i s^{6}$ is contained in the parabola $y=x^{2}$. We will show that there is a smooth first integral $W$ such that $W(\gamma(s))$ is not contained in a real analytic curve. Let $H$ be holomorphic in the semidisc $\{z=x+i y:|z|<1, y \geq 0\}$, smooth up to the $x$-axis, $H(0)=0, H^{\prime}(0) \neq 0$, and mapping the $x$-axis into a curve that is not real analytic in any neighborhood of the origin. The Riemann Mapping Theorem gives such maps. Let $W(x, t)=H(Z(x, t))$. Then $W$ is a smooth first integral of $L$ in a neighborhood of the origin. Observe that if $W(\gamma(s))=H\left(s^{3}+i s^{6}\right)$ is contained in a real analytic curve near $s=0$, then by the classical Schwarz Reflection Principle, $H$ would extend as a holomorphic function in a neighborhood of the parabola, and hence in a neighborhood of the origin, contradicting the hypothesis on $H$. 
We recall the concept of a reflection map $\sigma_{\Gamma}$ across a real analytic $\operatorname{arc} \Gamma$ of the complex plane:

Theorem (Theorem 9.1 in $[\mathbf{R}]$ ). If $\Gamma \subset \mathbb{C}$ is a connected real analytic arc, then there are a connected neighborhood $U$ of $\Gamma$ and a diffeomorphism $\sigma_{\Gamma}: U \rightarrow U$ such that the conjugate $\overline{\sigma_{\Gamma}}$ is holomorphic and $\sigma_{\Gamma}(z)=z$ for $z \in \Gamma$.

The following result gives a necessary condition for reflection across an $L$-analytic curve that is noncharacteristic to $L$ at a given point.

Theorem 1.1. Let $\gamma$ be an L-analytic smooth curve that is noncharacteristic to the smooth vector field $L$ at $0 \in \gamma$. Suppose $\gamma$ divides a disc $D$ centered at 0 into two components $D^{+}$and $D^{-}$, and $Z$ is a first integral of $L$ that maps $\gamma$ into a real analytic curve $\Gamma$. If any $u \in C\left(\overline{D^{+}}\right)$satisfying $L u=0$ in $D^{+}$which is real on $\gamma$ extends as a continuous solution in a neighborhood of 0 , then

(*) $\forall r_{1}>0, \exists r_{2}>0$ such that $Z\left(B_{r_{2}} \cap D^{-}\right) \subset Z\left(B_{r_{1}} \cap D^{+}\right) \cup \sigma_{\Gamma}\left(Z\left(B_{r_{1}} \cap D^{+}\right)\right)$.

Here $B_{r}$ denotes the disc centered at 0 of radius $r$.

Proof. Let $H$ be a local biholomorphism mapping $\Gamma$ into the real axis and $H(0)=0$. Let $F$ be a diffeomorphism on a neighborhood of the origin that sends 0 to 0 and maps the real axis into $\gamma$. Replacing $Z$ by $H \circ Z \circ F$ and using $x=\Re Z$ and $t$ as coordinates centered at the origin, we may assume that the first integral is $Z(x, t)=x+i \varphi(x, t)$ with $\varphi(x, 0) \equiv 0$. If condition $(\star)$ does not hold, then we may assume that for some positive number $a$, there are two sequences $z_{k}=x_{k}+i \varphi\left(x_{k}, t_{k}\right)$ and $\overline{z_{k}}=x_{k}-i \varphi\left(x_{k}, t_{k}\right)$ such that $\left(x_{k}, t_{k}\right) \rightarrow 0$ and for each $k, z_{k} \notin Z\left(Q^{+}\right)$and $\overline{z_{k}} \notin Z\left(Q^{+}\right)$, where $Q^{+}=[-a, a] \times[0, a]$. For each positive integer $k$, define

$$
f_{k}(x, t)=\frac{1}{\left(Z(x, t)-z_{k}\right)\left(Z(x, t)-\overline{z_{k}}\right)} .
$$

Each $f_{k}$ is real-valued on the $x$-axis, smooth away from $\left\{\left(x_{k}, t\right): t \leq 0\right\}$ and a solution on $\{(x, t) \in Q\} \backslash\left\{\left(x_{k}, t\right): t \leq 0\right\}$ where $Q=[-a, a] \times[-a, a]$. Assume first that only a finite number of the $x_{k}$ are zero, in which case, without loss of generality, we will assume that the $x_{k}$ decrease to zero. Let $d_{k}=\min \left(x_{k}-x_{k+1}, x_{k-1}-x_{k}\right) / 2$ for $k=2,3, \ldots$. Choose positive numbers $\alpha_{k}>0$ satisfying

$$
\begin{aligned}
& \left|\alpha_{k} f_{k}(x, t)\right| \leq 2^{-k} \text { for }(x, t) \in Q^{+} \text {and } \\
& \left|\alpha_{k} f_{k}(x, t)\right| \leq 2^{-k} \text { on }\left\{(x, t) \in Q:\left|x-x_{k}\right| \geq d_{k},|t| \leq a\right\} .
\end{aligned}
$$

Then the series

$$
f(x, t)=\sum_{k=1}^{\infty} \alpha_{k} f_{k}(x, t)
$$

converges uniformly on compact subsets of $W=Q \backslash \bigcup_{j=1}^{\infty}\{(x, t): t \leq 0, x=$ $x_{j}$ or $\left.x=0\right\}$, is a solution on this set, and is real-valued on the $x$-axis. Suppose now $w(x, t)$ is a continuous solution in a rectangle $V=(-b, b) \times(-b, b)$ that agrees with $f$ on $(-b, b) \times[0, b)$. Let $\left(x_{k}, t_{k}\right) \in V$ for $k>N$. Since $Z_{x}(0,0)=1$, if $b$ is small enough, since segments parallel to the $x$-axis are noncharacteristic, and $f$ is a solution on a connected subset of $V$, we have $w(x, t) \equiv f$ on $V \backslash W$. For $k>N$, observe that on the set $\left\{(x, t) \in V: x_{k}<x<x_{k}+d_{k}\right\}$, the function $f\left(x, t_{k}\right)$ is equal to $\alpha_{k} f_{k}\left(x, t_{k}\right)$ modulo a bounded function and hence $f(x, t)$ is unbounded on this set, which shows the nonexistence of an extension which is a continuous solution in any neighborhood of the origin. 
Suppose now only finitely many of the $x_{k}$ are nonzero. In that case we may assume that each $z_{k}=i \varphi\left(0, t_{k}\right)$, where the $t_{k}$ increase to 0 and the $\varphi\left(0, t_{j}\right)$ decrease strictly to 0 . For each $k$ define $f_{k}$ as before. Then each $f_{k}$ is a smooth solution away from $\{(0, t) \in Q: t \leq 0\}$. Choose positive numbers $\alpha_{k}$ such that

$$
\left|\alpha_{k} f_{k}(x, t)\right| \leq 2^{-k} \quad \text { on } Q^{+} \cup\left\{(x, t):|x| \geq \frac{a}{k}\right\} .
$$

We also require the $\alpha_{k}$ to satisfy

$$
\left|\alpha_{k} f_{k}\left(x, t_{j}\right)\right| \leq 2^{-k} \text { for } x \in[-a, a], j=1, \ldots, k-1 .
$$

Since $f\left(x, t_{k}\right)$ is unbounded as $x \rightarrow 0$, it follows that $f=\sum_{k} \alpha_{k} f_{k}$ does not have an extension that is a continuous solution in any neighborhood of the origin.

Next we will show that condition $(\star)$ is also sufficient.

Theorem 1.2. Let $L$ be a smooth, locally integrable vector field in a neighborhood of the origin and suppose $\gamma$ is a smooth, noncharacteristic L-analytic curve through the origin that divides a disc $D$ about $(0,0)$ into two regions $D^{+}$and $D^{-}$and $Z$ is a first integral of $L$ that maps $\gamma$ into a real analytic curve $\Gamma$. Assume that

(*) $\forall r_{1}>0, \exists r_{2}>0$ such that $Z\left(B_{r_{2}} \cap D^{-}\right) \subset Z\left(B_{r_{1}} \cap D^{+}\right) \cup \sigma_{\Gamma}\left(Z\left(B_{r_{1}} \cap D^{+}\right)\right)$.

If $u$ is continuous on $\overline{D^{+}}$such that $L u=0$ in $D^{+}$and $u(\gamma) \subset \sigma$ for some real analytic curve $\sigma$, then it extends as a continuous solution in a neighborhood of the origin.

Proof. We may assume that $u(\gamma) \subset \mathbb{R}$. Let $W$ be a smooth first integral of $L$ in a neighborhood of the origin such that $W(0)=0$ and $W(\gamma) \subset \Gamma$, where $\Gamma$ is a real analytic curve. Let $H$ be a local biholomorphism mapping $\Gamma$ into the real axis and $H(0)=0$. Let $F$ be a diffeomorphism on a neighborhood of the origin that sends 0 to 0 and maps the real axis into $\gamma$. Let $Z=H \circ W \circ F$. After composing with $F$ we may assume that $u(x, t)$ is a solution on the side $t>0$ (near the origin), continuous up to the $x$-axis where it is real-valued, and we wish to extend $u$ across the $x$-axis. Note that $Z(x, 0) \in \mathbb{R}$, and since the $x$-axis is noncharacteristic, the map $(x, t) \mapsto(\Re Z(x, t), t)$ is a change of coordinates in a neighborhood of the origin. We may therefore assume that $Z(x, t)=x+i \varphi(x, t)$, where $\varphi$ is real-valued, and $\varphi(x, 0) \equiv 0$. Hence, we may assume that $\Gamma$ is the $x$-axis and the map $\sigma_{\Gamma}$ is just complex conjugation. Given a rectangle $Q=[-a, a] \times[-c, d]$, we will write $Q^{+}=[-a, a] \times[0, d]$ and $Q^{-}=[-a, a] \times[-c, 0]$. Condition $(\star)$ allows us to find $a$, $c, d>0$, such that

$$
Z\left(Q^{-}\right) \subset Z\left(Q^{+}\right) \cup \sigma_{\Gamma}\left(Z\left(Q^{+}\right)\right)=Z\left(Q^{+}\right) \cup\{x-i \varphi(x, t):|x| \leq a, 0 \leq t \leq d\} .
$$

We may assume that $u$ is continuous on $\left[-a^{\prime}, a^{\prime}\right] \times[0, d]$ and a solution on $\left(-a^{\prime}, a^{\prime}\right) \times$ $(0, d)$ for some $a^{\prime}>a$. Assuming that $a>0$ and $d>0$ have been chosen small enough, we may apply the boundary version of the Baouendi-Treves approximation theorem and find a continuous function $U$ on $Z\left(Q^{+}\right)$that is holomorphic on the interior such that

$$
u(x, t)=U(Z(x, t)) \text { on } Q^{+} .
$$

For $|x| \leq a$, consider the Lipschitz continuous functions

$$
M_{1}(x)=\max _{0 \leq t \leq d} \varphi(x, t), \quad m_{1}(x)=\min _{0 \leq t \leq d} \varphi(x, t) .
$$


We may write

$$
\begin{aligned}
Z\left(Q^{+}\right) & =\left\{x+i y:|x| \leq a, m_{1}(x) \leq y \leq M_{1}(x)\right\}, \\
\sigma_{\Gamma}\left(Z\left(Q^{+}\right)\right) & =\left\{x-i y:|x| \leq a, m_{1}(x) \leq y \leq M_{1}(x)\right\} \\
& =\left\{x+i y:|x| \leq a,-M_{1}(x) \leq y \leq-m_{1}(x)\right\} .
\end{aligned}
$$

Since $\varphi(x, 0) \equiv 0, m_{1}(x) \leq 0 \leq M_{1}(x)$. Now consider the open set

$$
\Omega=\left\{x+i y:|x|<a, m_{1}(x)<y<M_{1}(x)\right\} \subset Z\left(Q^{+}\right) .
$$

Notice that $U(z)$ is holomorphic on $\Omega$, continuous on its closure $\bar{\Omega}$ and real for $z$ real. By the reflection principle, we may extend $U$ holomorphically to $\Omega^{0} \doteq \Omega \cup \Omega^{*}$ where

$$
\Omega^{*}=\left\{x-i y:|x|<a, m_{1}(x)<y<M_{1}(x)\right\}
$$

by setting $U(z)=\overline{U(\bar{z})}, z \in \Omega^{*}$, which is well defined. The same formula shows that, in fact, this holomorphic function has a continuous extension to the closed set

$$
K \cup K^{*}, \quad K=\left\{x+i y:|x| \leq a, m_{1}(x) \leq y \leq M_{1}(x)\right\} .
$$

We will denote by $U_{0}$ the extended function. Set $\mu(x)=\max \left(M_{1}(x),-m_{1}(x)\right)$, $|x| \leq a$, and notice that $-\mu(x)=\min \left(m_{1}(x),-M_{1}(x)\right)$. We have

$$
\begin{aligned}
& \Omega^{0} \doteq \Omega \cup \Omega^{*}=\{x+i y:|x|<a,|y|<\mu(x)\}, \\
& K^{0} \doteq K \cup K^{*}=\{x+i y:|x| \leq a,|y| \leq \mu(x)\} .
\end{aligned}
$$

Set $X=Q \cap Z^{-1}\left(K^{0}\right)$ and consider the continuous function $u_{0}(x, t)=U_{0}(Z(x, t))$, $(x, t) \in X$. Since $Z((-a, a) \times(0, d)) \subset K^{0}$, it follows that $(-a, a) \times(0, d) \subset X$, so also $(-a, a) \times(0, d) \subset \operatorname{Int} X$ (the interior of $X)$. We will prove the following facts:

(i) $\operatorname{Int} X=(-a, a) \times(-c, d)$;

(ii) $u_{0}(x, t)$ satisfies $L u_{0}=0$ on $(-a, a) \times(-c, d)$;

(iii) $u=u_{0}$ on $(-a, a) \times(0, d)$.

Once (i), (ii) and (iii) are proved, the proof of the theorem will be finished since $u_{0}(x, t)$ provides the desired extension of $u$ across $\gamma$. For $|x| \leq a$, consider the functions

$$
M_{2}(x)=\max _{-c \leq t \leq 0} \varphi(x, t), \quad m_{1}(x)=\min _{-c \leq t \leq 0} \varphi(x, t),
$$

in terms of which we may write

$$
Z\left(Q^{-}\right)=\left\{x+i y:|x| \leq a, m_{2}(x) \leq y \leq M_{2}(x)\right\} .
$$

The inclusion in $(\star)$ shows that $-\mu(x) \leq m_{2}(x) \leq 0 \leq M_{2}(x) \leq \mu(x)$. Then

$$
\mu(x)=0 \Longrightarrow \varphi(x, t)=0 \text { for }-c \leq t \leq d .
$$

Let $(x, t) \in[-a, a] \times[-c, d]$. Then

$$
-\mu(x) \leq \min \left(m_{1}(x), m_{2}(x)\right) \leq \varphi(x, t) \leq \max \left(M_{1}(x), M_{2}(x)\right) \leq \mu(x),
$$

so $Z(x, t) \in K^{0}$ and $(x, t) \in X$. This proves that $X=[-a, a] \times[-c, d]$ and shows that (i) holds.

By the Baouendi-Treves approximation formula, assuming that $a>0$ and $d>0$ were initially chosen conveniently small, the sequence of entire functions

$$
U_{j}(x+i y)=(j / \pi)^{1 / 2} \int_{\mathbb{R}} e^{-j\left(x+i y-x^{\prime}\right)^{2}} u\left(x^{\prime}, 0\right) h\left(x^{\prime}\right) d x^{\prime}, \quad j=1,2, \ldots,
$$


where $h\left(x^{\prime}\right) \in C_{c}^{\infty}\left(\left(-a^{\prime}, a^{\prime}\right)\right)$ is a test function that is identically equal to one on $[-2 a, 2 a]$, converges uniformly to $U_{0}(z)$ on $K=Z([0, a] \times[0, d])$. Since $u\left(x^{\prime}, 0\right)$ is real, $U_{j}(z)$ is real for $z$ real and we have $\bar{U}_{j}(z)=U_{j}(\bar{z}), z \in \mathbb{C}$. This implies that $U_{j}(z)$ converges uniformly to $U_{0}$ on $K \cup K^{*}=K^{0}$. Hence, $u_{j}(x, t)=U_{j}(Z(x, t))$ is a sequence of smooth solutions on $X$ that converge uniformly to $u_{0}(x, t)$ on $X$, showing that $u_{0}(x, t)$ is a weak solution on $(-a, a) \times(-c, d)$. This proves (ii).

To prove (iii), we observe that $L\left(u-u_{0}\right)=0$ on $(-a, a) \times(0, d)$ and $u(x, 0)-$ $u_{0}(x, 0) \equiv 0$ on $-a<x<a$ because of (i) and the definition of $u_{0}$. By uniqueness in the Cauchy problem, we have that $u(x, t) \equiv u_{0}(x, t)$ on $(-a, a) \times(0, d)$.

Next we consider the semilinear case for real analytic vector fields.

Theorem 1.3. Let $L$ be a real analytic vector field in a neighborhood of the origin and suppose $\gamma$ is a real analytic, noncharacteristic curve through the origin that divides a disc $D$ centered at $(0,0)$ into two regions $D^{+}$and $D^{-}$. Let $f(x, y, \zeta)$ be a real analytic function that is holomorphic in $\zeta$. Then if $u(x, y)$ is a continuous function on $\overline{D^{+}}$which is a solution of $L u=f(x, y, u)$ in $D^{+}$, and $u(\gamma)$ lies in a real analytic curve $\Gamma$, then $u$ extends as a solution of the same equation in a neighborhood of the origin.

Proof. Let $F_{1}(z)$ be a biholomorphism with $F_{1}(0)=0$ and mapping an interval $(-r, r)$ onto an open subarc of $\gamma$, and let $F_{2}$ be a biholomorphism with $F_{2}(u(0,0))=$ 0 that maps a neighborhood of $\Gamma$ about $u(0,0)$ into an interval $\left(-r^{\prime}, r^{\prime}\right)$. By replacing $u$ by $F_{2}^{-1} \circ u \circ F_{1}$, we may assume that $D^{+}=(-B, B) \times(0, A)$ and $u(x, 0) \in \mathbb{R}$. Let $Z(x, y)=x+i \varphi(x, y)$ be a real analytic first integral of $L$ in a neighborhood of the origin such that $\varphi(x, 0) \equiv 0$ and $\varphi$ is real-valued. If $\varphi$ is not identically zero, we can find an integer $m \geq 1$ and a real analytic function $\psi(x, y)$ such that $\varphi(x, y)=x^{m} \psi(x, y)$ with $\psi(0, y)$ not vanishing identically. For some $0<T<A$, we can assume that $|\psi(0, T)|>0$ and choose $\delta>0$ such that $|\psi(x, T)|>\frac{|\psi(0, T)|}{2}$ for $|x| \leq \delta$. It is not difficult to see that this implies that condition $(\star)$ is satisfied. The condition is also satisfied if $\varphi(x, y) \equiv 0$.

Let $G(x, y, \zeta)$ be the solution of the Cauchy problem

$$
\begin{aligned}
\frac{\partial G}{\partial y}+a(x, y) \frac{\partial G}{\partial x}+f(x, y, \zeta) \frac{\partial G}{\partial \zeta} & =0, \\
G(x, 0, \zeta) & =\zeta
\end{aligned}
$$

for $(x, y)$ near $(0,0)$ and $\zeta$ near $u(0,0)$. The function $G$ is real analytic. By inverting the map

$$
(z, w, \zeta) \longmapsto(z, w, G(z, w, \zeta))
$$

near $(0,0, u(0,0))$ in $\mathbb{C}^{3}$, we get a real analytic function $H(x, y, \eta)$ defined near $(0,0, u(0,0))$ such that

$$
H(x, y, G(x, y, \zeta))=\zeta
$$

In particular,

$$
u(x, y)=H\left(x, y, G(x, y, u(x, y)) \quad \text { on } D^{+} \text {after shrinking } D^{+} .\right.
$$

Define $w(x, y)=G(x, y, u(x, y))$ on $D^{+}$. Then $u(x, y)=H(x, y, w(x, y))$ on $D^{+}$ and by using the equations that $G$ and $u$ satisfy, we get

$$
L w=0 \text { in } D^{+} \text {and } w(x, 0)=u(x, 0) \in \mathbb{R} .
$$


Since condition $(\star)$ is satisfied, by Theorem 1.2 , there is $\delta>0$ and a continuous function $v(x, y)$ on $Q_{\delta}=(-\delta, \delta) \times(-\delta, \delta)$ that satisfies $L v=0$ in $Q_{\delta}$ and $v \equiv w$ on $Q_{\delta}^{+}$. Define

$$
\tilde{u}(x, y)=H(x, y, v(x, y)) \text { on } Q_{\delta} .
$$

Clearly, $\tilde{u}(x, y)=H(x, y, w(x, y))=u(x, y)$ on $Q_{\delta}^{+}$. On the rectangle $Q_{\delta}$, we have

$$
\begin{aligned}
L \tilde{u}(x, y) & =\frac{\partial H}{\partial y}(x, y, v)+\frac{\partial H}{\partial \eta}(x, y, v) \frac{\partial v}{\partial y}+a(x, y)\left(\frac{\partial H}{\partial x}+\frac{\partial v}{\partial x} \frac{\partial H}{\partial \eta}\right) \\
& =\frac{\partial H}{\partial y}(x, y, v)+\frac{\partial H}{\partial \eta}(x, y, v)\left(\frac{\partial v}{\partial y}+a(x, y) \frac{\partial v}{\partial x}\right)+a(x, y) \frac{\partial H}{\partial x} \\
& =\frac{\partial H}{\partial y}(x, y, v)+a(x, y) \frac{\partial H}{\partial x}(x, y, v)+\frac{\partial H}{\partial \eta}(L v) \\
& =\frac{\partial H}{\partial y}(x, y, v)+a(x, y) \frac{\partial H}{\partial x}(x, y, v) .
\end{aligned}
$$

Next we will show that $L H(x, y, v)=f(x, y, \tilde{u})$. Differentiating the equation $H(x, y, G(x, y, \zeta))=\zeta$ in $x$ and $y$, we get

$$
\begin{aligned}
& H_{y}(x, y, G)+H_{\eta}(x, y, G) G_{y}(x, y, \zeta)=0, \\
& H_{x}(x, y, G)+H_{\eta}(x, y, G) G_{x}(x, y, \zeta)=0 .
\end{aligned}
$$

Multiplying the second equation by $a(x, y)$ and adding it to the first equation yields

$$
\begin{aligned}
0 & =H_{y}(x, y, G)+a(x, y) H_{x}(x, y, G)+H_{\eta}(x, y, G)\left(G_{y}(x, y, \zeta)+a(x, y) G_{x}(x, y, \zeta)\right) \\
& =H_{y}(x, y, G)+a(x, y) H_{x}(x, y, G)-f(x, y, \zeta) G_{\zeta}(x, y, \zeta) H_{\eta}(x, y, G(x, y, \zeta)) .
\end{aligned}
$$

Since $G_{\zeta}(x, y, \zeta) H_{\eta}(x, y, G(x, y, \zeta))=1$, it follows that

$$
H_{y}(x, y, G)+a(x, y) H_{x}(x, y, G)-f(x, y, \zeta)=0 .
$$

Plugging $\zeta=H(x, y, v)$ in the latter equation and noting that $G(x, y, H(x, y, \eta))=$ $\eta$, we get

$$
\left.L \tilde{u}=H_{y}(x, y, v)+a(x, y) H_{x}(x, y, v)=f(x, y, H(x, y, v))=f(x, y, \tilde{u})\right) .
$$

Let $L=a(x, y) \partial_{x}+b(x, y) \partial_{y}$ be a not necessarily locally integrable vector field with smooth coefficients and assume that $L$ does not vanish on the domain $\Omega \subset \mathbb{R}^{2}$ where it is defined. We recall that $L$ is said to be hypoelliptic if for every distribution $u \in \mathcal{D}^{\prime}(\Omega)$, the singular supports of $u$ and $L u$ are equal. It is well known that if $L$ is hypoelliptic it must be locally solvable - therefore locally integrable - and hypocomplex.

Corollary 1.4. The reflection property always holds across noncharacteristic, $L$ analytic curves whenever $L$ is either a real analytic or a smooth, hypoelliptic vector field.

\section{The CHARACTERISTIC REFLECTION PROBLEM}

We now study the reflection problem for continuous solutions of a smooth locally integrable vector field $L$ across a characteristic $L$-analytic curve $\gamma$ that passes through the origin, i.e., $\gamma$ is an embedding of $[0,1]$ (in particular, $\gamma^{\prime}(s) \neq 0$ ) and $\gamma\left(s_{0}\right)=0$ for some $0<s_{0}<1$. We will assume that $\gamma^{\prime}\left(s_{0}\right)$ is a characteristic direction. 
We first consider the case where $\gamma$ is characteristic to $L$ at each of its points. When this happens we will say that $\gamma$ is totally characteristic. We may find local coordinates $(x, t),|x|<a,|t|<a$, in which $\gamma(s)$ is a subarc of the $t$-axis and $L$ has the form $L=\partial / \partial t+a(x, t) \partial / \partial x$ with $a(0, t) \equiv 0$. The next proposition shows that in this situation we may always extend solutions across $\gamma$.

Proposition 2.1. Let $L=\partial / \partial t+a(x, t) \partial / \partial x$ be a $C^{1}$ complex vector field in the rectangle $(-R, R) \times(-R, R)$. Assume that $f$ is continuous on $[0, R) \times(-R, R)$ and satisfies $L f=0$ in $(0, R) \times(-R, R)$. Suppose $a(0, t) \equiv 0$. Then $f$ extends as a continuous solution in a neighborhood of the origin.

Proof. Let $\phi \in C_{0}^{1}(-1,1)$ such that $\int \phi(x) d x=1$. For $\epsilon>0$ sufficiently small, set

$$
\psi_{\epsilon}(x)=\frac{1}{\epsilon} \phi\left(\frac{x-2 \epsilon}{\epsilon}\right) .
$$

Using the fact that $L f=0$ and integration by parts, for any $-R<\alpha<\beta<R$, we have

$$
\int_{\epsilon}^{3 \epsilon} f(x, \beta) \psi_{\epsilon}(x) d x-\int_{\epsilon}^{3 \epsilon} f(x, \alpha) \psi_{\epsilon}(x) d x=-\int_{\alpha}^{\beta} \int_{\epsilon}^{3 \epsilon} f(x, t) L^{t} \psi_{\epsilon}(x) d x d t,
$$

where $L^{t}$ denotes the transpose of $L$. Note that the left hand side converges to $f(0, \beta)-f(0, \alpha)$ as $\epsilon \rightarrow 0$. Write

$$
\begin{array}{r}
\int_{\alpha}^{\beta} \int_{\epsilon}^{3 \epsilon} f(x, t) L^{t} \psi_{\epsilon}(x) d x d t=\int_{\alpha}^{\beta} \int_{\epsilon}^{3 \epsilon}(f(x, t)-f(0, t)) L^{t} \psi_{\epsilon}(x) d x d t \\
+\int_{\alpha}^{\beta} \int_{\epsilon}^{3 \epsilon} f(0, t) L^{t} \psi_{\epsilon}(x) d x d t .
\end{array}
$$

Observe that

$$
\int_{\alpha}^{\beta} \int_{\epsilon}^{3 \epsilon} f(0, t) L^{t} \psi_{\epsilon}(x) d x d t=-\int_{\alpha}^{\beta} f(0, t)\left(\int_{\epsilon}^{3 \epsilon} \frac{\partial}{\partial x}\left(a(x, t) \psi_{\epsilon}(x)\right) d x\right) d t=0,
$$

since the support of $\psi_{\epsilon}$ is in $(\epsilon, 3 \epsilon)$. Next note that

$$
\begin{aligned}
\mid \int_{\alpha}^{\beta} \int_{\epsilon}^{3 \epsilon}(f(x, t) & -f(0, t)) L^{t} \psi_{\epsilon}(x) d x d t \mid \\
\leq & \left|\int_{\alpha}^{\beta} \int_{\epsilon}^{3 \epsilon}(f(x, t)-f(0, t)) \frac{\partial a}{\partial x} \psi_{\epsilon}(x) d x d t\right| \\
& \quad+\left|\int_{\alpha}^{\beta} \int_{\epsilon}^{3 \epsilon}(f(x, t)-f(0, t)) a(x, t) \frac{d}{d x} \psi_{\epsilon}(x) d x d t\right| \\
\leq & C\left(\omega(\epsilon)+\frac{\omega(\epsilon)}{\epsilon^{2}} \int_{\epsilon}^{3 \epsilon} x d x\right) \\
\leq & C_{1} \omega(\epsilon),
\end{aligned}
$$

where in the second inequality we have used the vanishing of $a(0, t)$ and used the notation $\omega(\epsilon)=\sup |f(x, t)-f(0, t)|$ on $[\epsilon, 3 \epsilon] \times[\alpha, \beta]$. Since $\omega(\epsilon) \rightarrow 0$, it follows that $f(0, t)$ is constant. If we now define

$$
g(x, t)= \begin{cases}f(x, t), & \text { for } x \geq 0 \\ f(0,0), & \text { for } x<0\end{cases}
$$

then it can easily be seen that $g$ is a solution in a neighborhood of the origin. 
Next we will consider reflection across the subclass of $L$-analytic curves which are zero sets of the real parts of first integrals with nonvanishing differential. Observe that at each point $p$ on such a curve, unless the vector field is elliptic at $p$, the curve is characteristic to the vector field. The following result gives a necessary condition for reflection across such curves.

Theorem 2.2. Let $L$ be a smooth, locally integrable vector field and let $\gamma$ be a curve through the origin given locally as $\gamma=\{(x, y): \Re Z(x, y)=0\}$, where $Z$ is a first integral of $L$ with $d(\Re Z) \neq 0$. Assume that no open subarc of $\gamma$ containing 0 is totally characteristic. Suppose $\gamma$ divides a disc $D$ centered at 0 into two components $D^{+}$and $D^{-}$, and set $\Gamma(s)=i$ s and notice that $Z(\gamma) \subset \Gamma$ as sets. If any $u \in C\left(\overline{D^{+}}\right)$ satisfying $L u=0$ in $D^{+}$which is real on $\gamma$ extends as a continuous solution in a neighborhood of 0 , then

$$
\forall r_{1}>0, \exists r_{2}>0 \text { such that } \sigma_{\Gamma}\left(Z\left(B_{r_{2}} \cap D^{-}\right)\right) \subset Z\left(B_{r_{1}} \cap D^{+}\right) .
$$

Here $B_{r}$ denotes the disc centered at 0 of radius $r$.

Proof. We may assume that we are in coordinates $(x, t)$ centered at the origin where the first integral takes the form $Z(x, t)=x+i \varphi(x, t)$ with $\varphi$ real-valued, $\varphi(0,0)=\varphi_{x}(0,0)=\varphi_{t}(0,0)=0$ and $\gamma(s)$ has the parametrization $\gamma(s)=(0, s)$. We may also assume that $D^{-}$is contained in $x<0, \Gamma$ is a subarc of the imaginary axis around the origin and the map $\sigma_{\Gamma}$ is just the reflection map $z=x+i y \mapsto-x+i y$.

Set $Q=(-a, a) \times[-a, a], Q^{+}=(0, a) \times[-a, a], Q^{-}=(-a, 0) \times[-a, a]$ and take $a>0$ small enough so that $Q \subset D$. The set $Z(Q)$ is given by

$$
Z(Q)=\{x+i y: \quad-a<x<a, \quad m(x) \leq y \leq M(x)\},
$$

where

$$
m(x)=\min _{-a \leq t \leq a} \varphi(x, t), \quad M(x)=\max _{-a \leq t \leq a} \varphi(x, t), \quad-a<x<a,
$$

are Lipschitz continuous functions. Since $\{0\} \times(-a, a)$ contains elliptic points arbitrarily close to the origin, we may assume after shrinking $a$, that $M(x)>m(x)$ for $x \in(-a, a)$. Let us point out an important consequence: $Q$ is a single Sussmann open orbit of $L$ relative to $Q$, so a solution of $L u=0$ is completely determined on $Q$ by its restriction to $Q^{+}$.

Let us assume that $(\star \star)$ does not hold. This implies that either $m(0)=0$ or $M(0)=0$; otherwise $Z(0)$ would be an interior point of $Z(Q)$ and condition $(\star \star)$ would hold. Assume first that $m(0)=0$, so $M(0)>0$, and we may assume that $\inf _{|x| \leq a} M(x)>0$. Note that for any $\varepsilon>0$ the inequality $m(x) \leq \inf _{|t| \leq \varepsilon} \varphi(-x, t)$ cannot hold on $0 \leq x \leq \varepsilon$, since this would imply $(\star \star)$. Thus, there exist sequences of numbers $\varepsilon_{k} \searrow 0, x_{k} \searrow 0$ and $t_{k} \rightarrow 0$ such that

$$
\varphi\left(-x_{k}, t_{k}\right)=\inf _{|t| \leq \varepsilon_{k}} \varphi\left(-x_{k}, t\right)<m\left(x_{k}\right), \quad k=1,2, \ldots
$$

Hence, setting $y_{k}=\varphi\left(-x_{k}, t_{k}\right), z_{k}=x_{k}+i y_{k}$ and $z_{k}^{\prime}=-x_{k}+i y_{k}$, we have

- $z_{k}, z_{k}^{\prime} \rightarrow 0$ as $k \rightarrow \infty$;

- $z_{k} \notin Z\left(Q^{+}\right)$and $z_{k}^{\prime} \in Z\left(Q^{-}\right)$for $k \in \mathbb{N}$. 
Then

$$
f_{k}(x+i y)=\frac{1}{\left(y-y_{k}-i\left(x-x_{k}\right)\right)\left(y-y_{k}-i\left(x+x_{k}\right)\right)}
$$

is a meromorphic function with simple poles precisely at $z=z_{k}$ and $z=z_{k}^{\prime}$ and is real on the imaginary axis. Hence, it is holomorphic on $Z\left(Q^{+}\right)$, has a pole on $Z\left(Q^{-}\right)$and, in particular, $F_{k}=f_{k} \circ Z(x, t)$ is a homogeneous solution of the vector field $L=\partial_{t}-\left(Z_{t} / Z_{x}\right) \partial_{x}$ on $Q^{+}$which is continuous on $\gamma$ and blows up at the point $\left(-x_{k}, t_{k}\right) \in Q^{-}$.

We will next construct a real sequence $a_{k} \searrow 0$ such that the series

$$
F(x, t)=\sum_{j=1}^{\infty} a_{j} F_{j}(x, t)
$$

defines a continuous function on $Q^{+}$that satisfies the equation $L F=0$ on $Q^{+}$, is continuous up to $x=0$, is real on $x=0$, but cannot be extended as a continuous (or even bounded) solution across the imaginary axis on any neighborhood of the origin. This will prove the theorem when $m(0)=0$.

For small $a>0$ the set $Z(Q)$ will be contained in the unit disc, and it will suffice to study the convergence of the series $\sum_{j} a_{j} f_{j}(z)$ for $|z|=|x+i y| \leq 1$. Assume without loss of generality that $\left|z_{j+1}\right|<\left|z_{j}\right|=\left|z_{j}^{\prime}\right|<1 / 2, j=1,2, \ldots$, and set

$$
\delta_{j}=2\left|z_{j}\right|, \quad K_{j}=\left\{z \in \mathbb{C}: \quad \delta_{j} \leq|z| \leq 1\right\}, \quad M_{j}=\sup _{K_{j}}\left|f_{j}(z)\right|, j=1,2 \ldots
$$

Note that $z_{\ell}, z_{\ell}^{\prime} \notin K_{j}$ for $\ell \geq j$, so $0<M_{j}<\infty, 1 \leq j<\infty$. If we choose $a_{k}$ so that $0<a_{k}<M_{k}^{-1} 2^{-k}$, we see that for $k \geq j$,

$$
a_{k} \sup _{K_{j}}\left|f_{k}(z)\right| \leq a_{k} \sup _{K_{k}}\left|f_{k}(z)\right| \leq 2^{-k},
$$

so $\sum_{k>j} a_{k} f_{k}(z)$ converges uniformly on $K_{j}$, showing that for every fixed $j$, the series $\sum_{k \geq j} a_{k} f_{k}(z)$ defines a holomorphic function on $\delta_{j}<|z|<1$ and $f(z) \doteq$ $\sum_{k \geq 1} a_{k} f_{k}(z)$ is a meromorphic function on the punctured unit disc with poles precisely at $\left\{z_{k}, z_{k}^{\prime}, k=1,2, \ldots\right\}$. It is clear that $f(z)$ is real for $z=i y,-1<y<1$. Set

$$
d_{k}=\min \left(\operatorname{dist}\left(z_{k}, Z\left(Q^{+}\right)\right) \text {, dist }\left(z_{k}^{\prime}, Z\left(Q^{+}\right)\right)\right) .
$$

By the choice of $z_{k}$ and $z_{k}^{\prime}, d_{k}>0$. Hence, if we further demand that $0<a_{k} \leq$ $d_{k}^{2} 2^{-k}$, the series $\sum_{k} a_{k} f_{k}(z)$ converges uniformly on $Z\left(\overline{Q^{+}}\right)$and $f(z)$ is continuous on $Z\left(\overline{Q^{+}}\right)$. Thus, the function $F(x, t)=f(Z(x, t))$ is continuous for $0 \leq x \leq a$, $|t| \leq a$, is real on $\{0\} \times[-a, a]$ and satisfies $L F=0$ on $Q^{+}$. We next show that $F(x, t)$ cannot be extended as a solution across the $t$-axis in any neighborhood of the origin. Indeed, if $u(x, t)$ is a solution defined on a disc $B_{r}$ centered at the origin that coincides with $F(x, t)$ on $B_{r} \cap Q^{+}$, it must also coincide with $F(x, t)$ on $V \doteq\left(B_{r} \cap Q^{-}\right) \backslash \bar{S}$, where

$$
S=\bigcup_{k=1}^{\infty}\left\{\left(-x_{k}, t\right) \in Q^{-}: \varphi\left(-x_{k}, t\right)=y_{k}\right\},
$$

because $\left(B_{r} \cap Q\right) \backslash \bar{S}$ is a connected open set and $F(x, t)-u(x, t)$ is a solution on $\left(B_{r} \cap Q\right) \backslash \bar{S}$ that vanishes on $B_{r} \cap Q^{+}$, and therefore on all of $\left(B_{r} \cap Q\right) \backslash \bar{S}$. For large $k$, 
the point $\left(-x_{k}, t_{k}\right) \in B_{r}$ and $u(x, t)$ does not remain bounded as $(x, t) \rightarrow\left(-x_{k}, t_{k}\right)$, which contradicts the continuity of $u(x, t)$ on $B_{r}$. The proof of the case $M(0)=0$ is similar.

Example. Suppose $Z(x, t)=x+i\left(t^{2}+2 x t+x\right), Q^{+}=(0,1) \times(-1,1), Q^{-}=$ $(-1,0) \times(-1,1), L=\partial_{t}-\left(Z_{t} / Z_{x}\right) \partial_{x}$. Then $Z\left(Q^{+}\right)$has four sides, three of them are straight segments and the lower side is given by the inverted parabola

$$
y=-x(x-1), \quad 0<x<1 .
$$

Similarly, $Z\left(Q^{-}\right)$is bounded below by the same parabola. Notice that the parabola remains strictly below the $x$-axis for $-1<x<0$ and strictly above the $x$-axis for $0<x<1$. It is apparent that condition $(\star \star)$ does not hold. Hence, by Theorem 2.2 , there exists a continuous function $u \in C\left(\overline{Q^{+}}\right)$that satisfies $L u=0$ on $Q^{+}$and is real for $x=0$ but cannot be extended as a solution to a neighborhood of the origin.

In the next section, we will prove that condition $(\star \star)$ is also sufficient to extend continuous solutions across $\gamma$.

\section{The APPRoximation PROBLEM}

We now study the approximation problem for a continuous homogeneous solution $L u=0$ of a smooth locally integrable vector field $L$ defined on one side of a smooth curve $\gamma$ that passes through the origin, i.e., $\gamma\left(s_{0}\right)=0$ for some $0<s_{0}<1$, and $\gamma$ is an embedding of $[0,1]$. This study will be instrumental in proving the converse of Theorem 2.2.

We assume that $u$ is defined on a one-sided neighborhood $U^{+}$of the origin determined by $\gamma$ and suppose that $u \in C\left(U^{+} \cup \gamma\right)$. The approximation problem is to determine whether it is possible, shrinking $U$ if necessary, to approximate $u$ uniformly on $U^{+} \cup \gamma$ by polynomials in some first integral $Z$.

If $\gamma$ is noncharacteristic, this is always possible by the Baouendi-Treves approximation theorem (boundary version). If $\gamma$ is totally characteristic $u$ can be extended as a solution to a neighborhood of the origin by Proposition 2.1, and the standard version of the Baouendi-Treves approximation theorem gives the approximation of the extended solution to a full neighborhood of 0 . Hence, the remaining case is that of a curve passing through the origin, which is a characteristic point, and such that no subarc of $\gamma$ that contains the origin is totally characteristic. For a subclass of these curves, those considered in Theorem 2.2, we have:

Theorem 3.1. Let $L$ be a smooth, locally integrable vector field and let $\gamma$ be a curve through the origin given locally as $\gamma=\{(x, y): \Re Z(x, y)=0\}$ where $Z$ is a first integral of $L$ with $d(\Re Z) \neq 0$. Assume that no open subarc of $\gamma$ containing 0 is totally characteristic. Suppose $\gamma$ divides a disc $D$ centered at 0 into two components $D^{+}$and $D^{-}$. If $u \in C\left(\overline{D^{+}}\right)$satisfies $L u=0$ in $D^{+}$, there is a sequence $p_{k}(Z)$ of polynomials in $Z$ and a disc $D_{0} \subset D$ centered at the origin such that $p_{k}(Z) \rightarrow u$ uniformly on $\overline{D_{0}^{+}}$

Before dealing with the proof of Theorem 3.1 we will first state and prove a technical lemma. 
Lemma 3.1. Let $\varphi(t):[a, b] \longrightarrow \mathbb{R}$ be $a C^{1}$ function and $u(t):[a, b] \longrightarrow \mathbb{R} a$ continuous function such that

(i) if $\varphi^{\prime}(t) \equiv 0$ on $[x, y] \subset[a, b]$, then $u(x)=u(y)$;

(ii) if $\varphi^{\prime}(x) \varphi^{\prime}(y) \neq 0$ and $\varphi(x)=\varphi(y)$, then $u(x)=u(y), x, y \in(a, b)$;

(iii) $\varphi(a)=\varphi(b)$.

Then $u(a)=u(b)$.

Proof. Assume without loss of generality that $\varphi(a)=\varphi(b)=0$. Set

$$
a_{1}=\sup \left\{x \in[a, b]:\left.\varphi\right|_{[a, x]} \equiv 0\right\}, \quad b_{1}=\inf \left\{x \in[a, b]:\left.\varphi\right|_{[x, b]} \equiv 0\right\} .
$$

It is clear that $\varphi\left(a_{1}\right)=\varphi\left(b_{1}\right)=0$ and, by (i), we also have $u(a)=u\left(a_{1}\right)$ and $u\left(b_{1}\right)=u(b)$. Hence, renaming $a_{1}$ and $b_{1}$ as $a$ and $b$, there is no restriction in assuming from the start that $a, b \in \bar{U}$, where $U=\{x \in[a, b]: \varphi(x) \neq 0\}$. Set

$$
U^{+}=\{x \in U: \varphi(x)>0\}, \quad U^{-}=\{x \in U: \varphi(x)<0\} .
$$

Suppose first that $a, b \in \overline{U^{+}}$and take a sequence $\left(a_{j}\right), a_{j}>a, a_{j} \searrow a$ such that $\lambda_{j} \doteq \varphi\left(a_{j}\right)>0$ and $\varphi^{\prime}\left(a_{j}\right) \neq 0$. For large $j$ we may also find points $b_{j}<b, b_{j} \nearrow b$ such that $\varphi\left(b_{j}\right)=\lambda_{j}$. Should $b_{j}$ be contained in a maximal interval $\left[x_{j}, y_{j}\right]$ on which $\varphi^{\prime}(t) \equiv 0$, replace $b_{j}$ by $y_{j}$ and keep calling it $b_{j}$. For each $j$, let $0<\delta_{j}<\frac{1}{j}$ such that

$$
\left|\varphi(t)-\varphi\left(a_{j}\right)\right|<\frac{1}{j} \text { and } \varphi^{\prime}(t) \neq 0 \text { when }\left|t-a_{j}\right|<\delta_{j} .
$$

Fix $j$. Let $t_{k} \rightarrow b_{j}$ such that $\varphi^{\prime}\left(t_{k}\right) \neq 0$. Since $\varphi\left(t_{k}\right) \rightarrow \varphi\left(b_{j}\right)=\varphi\left(a_{j}\right)$, it is clear that we can find $a_{j}^{\prime}$ satisfying $\left|a_{j}^{\prime}-a_{j}\right|<\delta_{j}$ and $b_{j}^{\prime} \doteq t_{k}$ for some $k$ such that $\left|b_{j}^{\prime}-b_{j}\right|<\frac{1}{j}$ and $\varphi\left(a_{j}^{\prime}\right)=\varphi\left(b_{j}^{\prime}\right)$. Replace $a_{j}$ by $a_{j}^{\prime}$ and $b_{j}$ by $b_{j}^{\prime}$. Then (ii) implies $u\left(a_{j}\right)=u\left(b_{j}\right)$, and letting $j \rightarrow \infty$ we get $u(a)=u(b)$ in this case. A similar argument can be given for the case $a, b \in \overline{U^{-}}$.

Let us suppose that $a \in \overline{U^{-}}$and $b \in \overline{U^{+}}$(the case $a \in \overline{U^{+}}, b \in \overline{U^{-}}$is analogous). Suppose first that $U^{-}$has a connected component $(a, c)$ for some $c \in(a, b)$. Observing that $\varphi(t) \leq 0$ on $[a, c]$ and reasoning as above with the interval $[a, c]$ in place of $[a, b]$, we see that $u(a)=u(c)$. Replace $c_{1}$ by $c$ if there is a maximal interval $\left[c, c_{1}\right]$ on which $\varphi^{\prime}(t)$ vanishes identically, keep calling it $c$ and note that, by Proposition 2.1, we still have $u(c)=u(a)$. Now, focus on the interval $[c, b]$ and observe that it contains points $c_{j}$ arbitrary close to $c$ with $\varphi\left(c_{j}\right)>0$. We are now in the same situation considered at the beginning, when we assumed that $a, b \in \overline{U^{+}}$, and may conclude that $u(c)=u(b)$. Thus, $u(a)=u(b)$ in this case. If $U^{-}$does not have a connected component of the form $(a, c), c \in(a, b)$, there exists a sequence $\left(a_{j}, c_{j}\right)$ of connected components of $U^{-}$with $a_{j} \rightarrow a$. Reasoning with $\left(a_{j}, c_{j}\right)$ as we just did with $(a, c)$, we conclude that $u\left(a_{j}\right)=u(b)$, and letting $a_{j} \rightarrow a$ we get $u(a)=u(b)$.

Corollary 3.1. Let $\varphi(t):[a, b] \longrightarrow \mathbb{R}$ be a $C^{1}$ function, $u(t):[a, b] \longrightarrow \mathbb{R} a$ continuous function and set $M=\sup \varphi(t), m=\sup \varphi(t)$. Assume that

(i) when $\varphi^{\prime}(t) \equiv 0$ on $[x, y] \subset[a, b]$, then $u(x)=u(y)$;

(ii) if $\varphi^{\prime}(x) \varphi^{\prime}(y) \neq 0$ and $m<\varphi(x)=\varphi(y)<M$, then $u(x)=u(y), x, y \in$ $(a, b)$.

Then $\varphi(x)=\varphi(y)$ implies $u(x)=u(y), x, y \in[a, b]$. 
Proof. Since maxima and minima of $\varphi(t)$ that lie in $(a, b)$ are critical points, the requirement $m<\varphi(x)=\varphi(y)<M$ in (ii) imposes no additional restriction as compared with (ii) of Lemma 3.1. It is enough to apply Lemma 3.1 on the interval $[x, y]$ to reach the conclusion.

We now prove the theorem.

Proof. We may assume that $Z(x, t)=x+i \varphi(x, t), \varphi(0,0)=0, \gamma(s)=(0, s)$; in particular, $Z(\gamma)$ is contained in the imaginary axis.

Set $Q=[-a, a] \times[-a, a], Q^{+}=(0, a] \times[-a, a]$, and take $a>0$ small enough so that $Q \subset D$. The set $Z(Q)$ is given by

$$
Z(Q)=\{x+i y: \quad-a \leq x \leq a, \quad m(x) \leq y \leq M(x)\},
$$

where

$$
m(x)=\min _{-a \leq y \leq a} \varphi(x, y) \quad M(x)=\max _{-a \leq y \leq a} \varphi(x, y), \quad-a<x<a .
$$

Since $\{0\} \times(-a, a)$ contains elliptic points arbitrary close to the origin, we may assume after shrinking $a$ that $M(x)>m(x)$ for $x \in(-a, a)$. We will show that after shrinking $a>0$ we can find a holomorphic function $U^{+}(x+i y)$, defined on

$$
\Omega^{+}=\{x+i y: \quad 0<x<a, \quad m(x)<y<M(x)\}
$$

and continuous on

$$
\{x+i y: \quad 0<x \leq a, \quad m(x) \leq y \leq M(x)\},
$$

such that

$$
u(x, t)=U^{+}(Z(x, t)) \quad 0<x \leq a, m(x) \leq y \leq M(x) .
$$

We wish to first recall the integrals involved in the Baouendi-Treves approximation theorem. We may assume that $\left|\varphi_{x}(x, t)\right| \leq \frac{1}{2}$ on $[0, a] \times[-a, a]$. Let $u(x, t)$ be a continuous function on $[0, a] \times[-a, a]$ that is a solution in the interior. Fix an interval $[c, d] \subset(0, a)$. Let $g \in C_{0}^{\infty}(c, d)$ such that $g \equiv 1$ on $\left[c^{\prime}, d^{\prime}\right]$ for some $c<c^{\prime}<d^{\prime}<d$. For any $\tau$, using Stokes' theorem, one has for any $t \in[-a, a]$ (say $t>0)$

$$
\begin{array}{rl}
\frac{\tau}{\sqrt{\pi}} \int_{c}^{d} e^{-\tau^{2}\left(Z(x, t)-Z\left(x^{\prime}, t\right)\right)^{2}} & u\left(x^{\prime}, t\right) g\left(x^{\prime}\right) d Z\left(x^{\prime}, t\right) \\
& -\frac{\tau}{\sqrt{\pi}} \int_{c}^{d} e^{-\tau^{2}\left(Z(x, t)-Z\left(x^{\prime}, 0\right)\right)^{2}} u\left(x^{\prime}, 0\right) g\left(x^{\prime}\right) d Z\left(x^{\prime}, 0\right) \\
& =\frac{\tau}{\sqrt{\pi}} \int_{0}^{t} \int_{c}^{d} e^{-\tau^{2}\left(Z(x, t)-Z\left(x^{\prime}, t^{\prime}\right)\right)^{2}} u\left(x^{\prime}, t^{\prime}\right) d g \wedge d Z .
\end{array}
$$

The assumption that $\left|\varphi_{x}(x, t)\right| \leq 1 / 2$ guarantees that as $\tau \rightarrow \infty$, the first integral on the left converges uniformly to $g(x) u(x, t)$. The integral on the right will converge uniformy to zero if $|t| \leq \delta$ for some $\delta$ that depends only on the numbers $c^{\prime}-c, d-d^{\prime}$.

We may therefore assume that there are functions $U_{1}, U_{2}$, where $U_{1}$ is continuous on $Z\left(\left[c^{\prime}, d^{\prime}\right] \times[0, \delta]\right)$, holomorphic on the interior, $u(x, t)=U_{1}(Z(x, t))$ on $\left[c^{\prime}, d^{\prime}\right] \times$ $[0, \delta]$, and $U_{2}$ is continuous on $Z\left(\left[c^{\prime}, d^{\prime}\right] \times[\delta, 2 \delta]\right)$, holomorphic on the interior, with $u(x, t)=U_{2}(Z(x, t))$ on $\left[c^{\prime}, d^{\prime}\right] \times[\delta, 2 \delta]$.

Next we wish to show that $U_{1}$ and $U_{2}$ give rise to a function $U$ on the union of their domains that is holomorphic on the interior of $Z\left(\left[c^{\prime}, d^{\prime}\right] \times[0,2 \delta]\right)$. 
Let $x_{1} \in\left[c^{\prime}, d^{\prime}\right]$ and suppose $\varphi\left(x_{1}, t\right) \equiv$ constant on $[0, \delta]$. Then by Proposition 2.1, $U_{1}\left(Z\left(x_{1}, t\right)=u\left(x_{1}, \delta\right)\right.$ for all $0 \leq t \leq \delta$. On the other hand, if $Z\left(x_{1}, s\right)=$ $Z\left(x_{1}, \delta\right)$ for some $\delta<s \leq 2 \delta, U_{2}\left(Z\left(x_{1}, s\right)\right)=U_{2}\left(Z\left(x_{1}, \delta\right)\right)=U_{1}\left(Z\left(x_{1}, \delta\right)\right)$, and so the function defined by

$$
U\left(Z\left(x_{1}, t\right)\right)= \begin{cases}U_{1}\left(Z\left(x_{1}, \delta\right)\right), & 0 \leq t \leq \delta \\ U_{2}\left(Z\left(x_{1}, t\right)\right), & \delta \leq t \leq 2 \delta\end{cases}
$$

is well defined on $[0,2 \delta]$. The same conclusion holds if $\varphi\left(x_{1}, t\right)$ is constant on $[\delta, 2 \delta]$.

Define the set

$$
A=\left\{x \in\left(c^{\prime}, d^{\prime}\right): M_{2}(x)>m_{2}(x) \text { and } M_{1}(x)>m_{1}(x)\right\},
$$

where

$$
\begin{aligned}
& M_{1}(x)=\sup _{0 \leq t \leq \delta} \varphi(x, t), \quad m_{1}(x)=\inf _{0 \leq t \leq \delta} \varphi(x, t), \\
& M_{2}(x)=\sup _{\delta \leq t \leq 2 \delta} \varphi(x, t), \quad m_{2}(x)=\inf _{\delta \leq t \leq 2 \delta} \varphi(x, t) .
\end{aligned}
$$

Note that the set $A$ is open, $m_{2}(x) \leq \varphi(x, \delta) \leq M_{1}(x)$ and $m_{1}(x) \leq \varphi(x, \delta) \leq$ $M_{2}(x)$. Fix a component $I=\left(a_{k}, b_{k}\right)$ of $A$. Suppose $m_{2}(x)=M_{1}(x)$ at some $x \in I$. Then $m_{2}(x)=\varphi(x, \delta)=M_{1}(x)$, which implies that $U_{1}\left(x+i M_{1}(x)\right)=$ $U_{2}\left(x+i m_{2}(x)\right)$, and so if $\varphi\left(x, t_{1}\right)=\varphi\left(x, t_{2}\right)$ for some $t_{1} \in[0, \delta]$ and $t_{2} \in[\delta, 2 \delta]$, then the inequality

$$
\varphi\left(x, t_{1}\right) \leq M_{1}(x)=m_{2}(x) \leq \varphi\left(x, t_{2}\right)
$$

leads to $\varphi\left(x, t_{1}\right)=\varphi(x, \delta)=\varphi\left(x, t_{2}\right)$. It follows that

$$
U(Z(x, t))= \begin{cases}U_{1}(Z(x, t)), & 0 \leq t \leq \delta \\ U_{2}(Z(x, t)), & \delta \leq t \leq 2 \delta\end{cases}
$$

is well defined for $t \in[0,2 \delta]$. A similar conclusion is reached if we assume that $m_{1}(x)=M_{2}(x)$. Next consider a point $x_{0} \in I$ where $m_{2}\left(x_{0}\right)<M_{1}\left(x_{0}\right)$ and $m_{1}\left(x_{0}\right)<M_{2}\left(x_{0}\right)$. Let $J$ be an interval around $x_{0}$ such that

$$
m_{2}(x)<M_{1}(x) \text { and } m_{1}(x)<M_{2}(x) \forall x \in J .
$$

Then we have

$$
\begin{aligned}
\left\{x+i y: x \in J, m_{1}(x)<y<M_{1}(x)\right\} & \cap\left\{x+i y: x \in J, m_{2}(x)<y<M_{2}(x)\right\} \\
& =\{x+i y: x \in J, m(x)<y<M(x)\},
\end{aligned}
$$

where $m(x)=\max \left(m_{1}(x), m_{2}(x)\right)$ and $M(x)=\min \left(M_{1}(x), M_{2}(x)\right)$. Since each $U_{j}$ is continuous on the set $\left\{x+i y: x \in J, m_{j}(x) \leq y \leq M_{j}(x)\right\}(j=1,2)$, holomorphic on the interior and the $U_{j}$ agree on the curve $\{x+i \varphi(x, \delta): x \in J\}$ which is contained in the intersection

$$
\left\{x+i y: x \in J, m_{1}(x) \leq y \leq M_{1}(x)\right\} \cap\left\{x+i y: x \in J, m_{2}(x) \leq y \leq M_{2}(x)\right\},
$$

we conclude that $U_{1} \equiv U_{2}$ on

$$
\left\{x+i y: x \in J, m_{1}(x)<y<M_{1}(x)\right\} \cap\left\{x+i y: x \in J, m_{2}(x)<y<M_{2}(x)\right\} .
$$

This means that $U_{1}$ and $U_{2}$ define a holomorphic function on the union

$$
\left\{x+i y: x \in J, m_{1}(x)<y<M_{1}(x)\right\} \cup\left\{x+i y: x \in J, m_{2}(x)<y<M_{2}(x)\right\} .
$$


It follows that $U_{1}$ and $U_{2}$ give rise to a function $U$ that is continuous on $Z\left(\left(c^{\prime}, d^{\prime}\right) \times\right.$ $[0,2 \delta])$, holomorphic on the interior and $u(x, t)=U(Z(x, t))$ for $(x, t) \in\left[c^{\prime}, d^{\prime}\right] \times$ $[0,2 \delta]$. After repeating this procedure a finite number of times, we get a function $V$ that is continuous on $Z\left(\left[c^{\prime}, d^{\prime}\right] \times[-a, a]\right)$, holomorphic on the interior and satisfying $u(x, t)=V(Z(x, t))$ for $(x, t) \in\left[c^{\prime}, d^{\prime}\right] \times[-a, a]$. Let $0<c_{k}<d_{k}<a$ be two sequences such that $c_{k} \rightarrow 0$ and $d_{k} \rightarrow a$. We have shown that for each $k$, there is a function $V_{k}$ that is continuous on $Z\left(\left[c_{k}, d_{k}\right) \times[-a, a]\right)$ and holomorphic on the interior such that $u(x, t)=V_{k}(Z(x, t))$ on $\left[c_{k}, d_{k}\right] \times[-a, a]$. The $V_{k}$ give rise to a function $U^{+}$continuous on $Z((0, a) \times[-a, a])$, holomorphic on the interior and such that

$$
u(x, t)=U^{+}(Z(x, t)) \quad \text { for }(x, t) \in(0, a) \times[-a, a] .
$$

We wish to extend $U^{+}$continuously to

$$
\overline{\Omega^{+}}=\{x+i y: \quad 0 \leq x \leq a, \quad m(x) \leq y \leq M(x)\} .
$$

Once this is done, the theorem will easily follow by approximating the extended function $U^{+}$uniformly on $\overline{\Omega^{+}}$by a sequence of holomorphic polynomials $\left(p_{k}(z)\right)$ (which is possible by Mergelyan's theorem [M]). Noting that $u(x, t)=U(Z(x, t)$ ), $0 \leq x \leq a,-a \leq t \leq a$, this gives the required approximation of $u$ by $p_{k} \circ Z$ on $\overline{D_{0}^{+}}$ for any disc $D_{0} \subset Q$.

To extend $U^{+}(z)$ up to $x=0$ it will be enough, by a standard argument, to show that $u(x, t)$ is constant on the fibers of $Z$ over $[0, a] \times[-a, a]$. In other words, we must show that

$$
\varphi\left(x, t_{1}\right)=\varphi\left(x, t_{2}\right) \Longrightarrow u\left(x, t_{1}\right)=u\left(x, t_{2}\right) \quad 0 \leq x \leq a,-a \leq t \leq a .
$$

For any $0<x \leq a(3.1)$ implies (3.2), so we should only concern ourselves with the validity of $(3.2)$ for points of the form $(0, t)$. Let us consider two points $p_{1}=\left(0, t_{1}\right)$, $p_{2}=\left(0, t_{2}\right),-a \leq t_{1}<t_{2} \leq a$, such that $\varphi\left(0, t_{1}\right)=\varphi\left(0, t_{2}\right)$ and suppose that

$$
\left.\varphi_{t}\left(0, t_{1}\right) \neq 0, \varphi_{t}\left(0, t_{2}\right) \neq 0, m(0)<\varphi\left(0, t_{1}\right)=\varphi\left(0, t_{2}\right)\right) \doteq \lambda<M(0) .
$$

By the implicit function theorem, the level set

$$
\mathcal{C}_{\lambda}=\{(x, t): \quad \varphi(x, t)=\lambda\}
$$

contains smooth graphs $\left\{\left(x, t_{1}(x)\right): 0 \leq x<\epsilon\right\},\left\{\left(x, t_{2}(x)\right): 0 \leq x<\epsilon\right\} \subset \Omega^{+}$ passing respectively through the points $p_{1}$ and $p_{2}$. It follows that $u\left(x, t_{1}(x)\right)=$ $\lambda=u\left(x, t_{2}(x)\right)$ for $0<x<\varepsilon$, and letting $x \searrow 0$, we obtain $u\left(0, t_{1}\right)=u\left(0, t_{2}\right)$. We will apply Corollary 3.1 on the interval $[-a, a]$ to the functions $t \mapsto \varphi(0, t)$ and $t \mapsto u(0, t)$. We have just shown that the hypothesis (ii) is fulfilled while the validity of (i) follows from an application of Proposition 2.1. Therefore, $u(0, t)$ is constant on the fibers of $\varphi(0, t)$ on $[-a, a]$. This implies that $u(x, t)$ is constant on the fibers of $Z(x, t)$ over $((0, a] \times[-a, a]) \cup(\{0\} \times V)$. This allows us to continuously extend $U^{+}(x+i y)$ to

$$
\{x+i y: \quad 0<x \leq a, m(x) \leq y \leq M(x)\} \cup\{i y: \quad m(0)<y<M(0)\}
$$

by setting $U^{+}(i y)=u(0, t)$ for any $-a \leq t \leq a$ such that $\varphi(0, t)=y$.

We will next use Theorem 3.1 to show that the reflection property holds when condition $(\star \star)$ is satisfied. 
Corollary 3.2. Let $L$ be a smooth, locally integrable vector field and $\gamma$ be a curve through the origin given locally by $\gamma=\{(x, y): \Re Z(x, y)=0\}$, where $d(\Re Z) \neq 0$. Suppose that near the origin, $u$ is a continuous solution of $L u=0$ on a side of $\gamma$ and continuous up to $\gamma$. If $u(\gamma) \subset \sigma$ for some real analytic curve $\sigma$ and condition ( $\star \star)$ holds, then it can be extended as a continuous solution to a neighborhood of the origin.

Proof. Observe that if $\gamma$ is characteristic to $L$ at every point in a neighborhood of 0 , then by Proposition 2.1, $u$ extends as a continuous solution to a neighborhood of the origin. We may therefore assume that the origin is the limit of a sequence of noncharacteristic points in $\gamma$ for $L$. We will continue to use the special coordinates $(x, t),|x|,|t| \leq a$, the first integral $Z(x, t)=x+i \varphi(x, t)$ and $\gamma(s)=(0, s)$ considered in Theorem 3.1. By Theorem 3.1, we may find a function $U^{+}(x+i y)$, holomorphic on

$$
\Omega^{+}=\{x+i y: \quad 0<x<a, \quad m(x)<y<M(x)\}
$$

and continuous on

$$
\{x+i y: \quad 0 \leq x \leq a, \quad m(x) \leq y \leq M(x)\},
$$

such that

$$
u(x, t)=U^{+}(Z(x, t)) \quad 0 \leq x \leq a, m(x) \leq y \leq M(x) .
$$

Since $U^{+}$sends $x=0$ into $\sigma$, it can be extended to a holomorphic function $U$ by the Schwarz Reflection Principle. Condition ( $\star \star)$ implies that the composition $U \circ Z$ is defined in a neighborhood of the origin and extends $u$. By Mergelyan's theorem $\left[\mathrm{M}\right.$, there is a sequence of polynomials $p_{k}(z)$ that converges uniformly to $U(z)$, and hence $h(x, t)=U(Z(x, t))$ is a solution of $L h=0$ in a neighborhood of the origin that extends $u$.

\section{REFERENCES}

[BER] M. S. Baouendi, P. Ebenfelt, and L. Rothschild, Mappings between real submanifolds in complex space, Princeton University Press, 1995.

[BGT] M. S. Baouendi, C. Goulaouic, and F. Trèves, Uniqueness in certain first-order nonlinear complex Cauchy problems, Comm. Pure Appl. Math. 38 (1985), no. 1, 109-123, DOI 10.1002/cpa.3160380106. MR.768106 (86g:35045)

[BJT] M. S. Baouendi, H. Jacobowitz, and F. Trèves, On the analyticity of CR mappings, Ann. of Math. (2) 122 (1985), no. 2, 365-400, DOI 10.2307/1971307. MR808223 (87f:32044)

[BHR] M. S. Baouendi, Xiaojun Huang, and Linda Preiss Rothschild, Regularity of CR mappings between algebraic hypersurfaces, Invent. Math. 125 (1996), no. 1, 13-36, DOI 10.1007/s002220050067. MR1389959 (97c:32028)

[BR] M. S. Baouendi and Linda Preiss Rothschild, A general reflection principle in $\mathbf{C}^{2}$, J. Funct. Anal. 99 (1991), no. 2, 409-442, DOI 10.1016/0022-1236(91)90047-9. MR1121620 (92j:32035)

[BH] S. Berhanu and J. Hounie, On boundary properties of solutions of complex vector fields, J. Funct. Anal. 192 (2002), no. 2, 446-490, DOI 10.1006/jfan.2001.3914. MR1923410 (2004i:31006)

$[\mathrm{BCH}]$ Shiferaw Berhanu, Paulo D. Cordaro, and Jorge Hounie, An introduction to involutive structures, New Mathematical Monographs, vol. 6, Cambridge University Press, Cambridge, 2008. MR2397326 (2009b:32048)

[DF] K. Diederich and J. E. Fornæss, Proper holomorphic mappings between real-analytic pseudoconvex domains in $\mathbf{C}^{n}$, Math. Ann. 282 (1988), no. 4, 681-700, DOI 10.1007/BF01462892. MR:970228 (89m:32045) 
[EH] Peter Ebenfelt and Xiaojun Huang, On a generalized reflection principle in $\mathbb{C}^{2}$, Complex analysis and geometry (Columbus, OH, 1999), Ohio State Univ. Math. Res. Inst. Publ., vol. 9, de Gruyter, Berlin, 2001, pp. 125-140. MR1912734 (2003e:32019)

[Hu1] Xiaojun Huang, Schwarz reflection principle in complex spaces of dimension two, Comm. Partial Differential Equations 21 (1996), no. 11-12, 1781-1828, DOI 10.1080/03605309608821246. MR:1421212 (97m:32043)

[Hu2] Xiaojun Huang, On some problems in several complex variables and CR geometry, First International Congress of Chinese Mathematicians (Beijing, 1998), AMS/IP Stud. Adv. Math., vol. 20, Amer. Math. Soc., Providence, RI, 2001, pp. 383-396. MR1830195 (2002b:32001)

[M] S. N. Mergelyan, Uniform approximations of functions of a complex variable, Uspehi Matem. Nauk (N.S.) 7 (1952), no. 2(48), 31-122 (Russian). MR0051921 (14,547e)

[Me] Francine Meylan, A reflection principle in complex space for a class of hypersurfaces and mappings, Pacific J. Math. 169 (1995), no. 1, 135-160. MR.1346250 (96f:32018)

[R] R. Michael Range, Holomorphic functions and integral representations in several complex variables, Graduate Texts in Mathematics, vol. 108, Springer-Verlag, New York, 1986. MR.847923 (87i:32001)

[S] H. A. Schwarz, Uber einige Abbildungsaufgaben, J. Reine Angew. Math. 70 (1869), 105120.

[T] François Trèves, Hypo-analytic structures, Princeton Mathematical Series, vol. 40, Princeton University Press, Princeton, NJ, 1992. Local theory. MR.1200459 (94e:35014)

Department of Mathematics, Temple University, Philadelphia, Pennsylvania 191226094

E-mail address: berhanu@temple.edu

Departamento de Matemática, University Federal de São Carlos, 13.565-905, São CARlos, SP, BraziL

E-mail address: hounie@dm.ufscar.br 